Tohoku Math. J

56 (2004), 125-145

\title{
ON THE FOURIER COEFFICIENTS OF MODULAR FORMS \\ OF HALF INTEGRAL WEIGHT BELONGING TO KOHNEN'S SPACES AND THE CRITICAL VALUES OF ZETA FUNCTIONS
}

\author{
HisAshi KoJIMA AND YASUSHI TOKUNO
}

(Received April 4, 2002, revised September 22, 2003)

\begin{abstract}
The purpose of this paper is to derive a generalization of Kohnen-Zagier's results concerning Fourier coefficients of modular forms of half integral weight belonging to Kohnen's spaces, and to refine our previous results concerning Fourier coefficients of modular forms of half integral weight belonging to Kohnen's spaces. Employing kernel functions, we construct a correspondence $\Psi$ from modular forms of half integral weight $k+1 / 2$ belonging to Kohnen's spaces to modular forms of weight $2 k$. We explicitly determine the Fourier coefficients of $\Psi(f)$ in terms of those of $f$. Moreover, under certain assumptions about $f$ concerning the multiplicity one theorem with respect to Hecke operators, we establish an explicit connection between the square of Fourier coefficients of $f$ and the critical value of the zeta function associated with the image $\Psi(f)$ of $f$ twisted with quadratic characters, which gives a further refinement of our results concerning Fourier coefficients of modular forms of half integral weight belonging to Kohnen's spaces.
\end{abstract}

Introduction. In [20], Waldspurger first found the proportionality between Fourier coefficients of modular forms of half integral weight and special values of twisted $L$-series defined by Shimura correspondences. Kohnen-Zagier [4], [6] and [7] determined explicitly the constant of proportionality in the case of modular forms of half integral weight belonging to Kohnen's spaces of odd level and of trivial character.

In [17], Shimura proved many general formulas of Waldspurger-type in the case of Hilbert modular forms $f$ of half integral weight. Among these, some explicit and useful formulas about the proportionality constant were formulated under assumption that $f$ satisfies the multiplicity one theorem of Hecke operators. Manickam-Ramakrishnan-Vasudevan [13] and [14] also obtained related results in the case of elliptic modular forms of half integral weight. On the other hand, Kojima [8] and [9] generalized Kohnen-Zagier's results to the case of Kohnen's spaces of arbitrary odd level and of arbitrary character under the assumption of multiplicity two theorem of Hecke operators. It is to be desired that, under the assumption of multiplicity one theorem, our results are reformulated in the case of arbitrary odd level and of arbitrary character in conjunction with the possibility of a further refinement of [8] and [9].

2000 Mathematics Subject Classification. Primary 11F 37; Secondary 11F30, 11F67.

Key words and phrases. Modular forms of half integral weight, Fourier coefficients of modular forms, special value of zeta function. 
We refer to [10] and [12] for a generalization of Shimura's results to the case of Maass wave forms.

The purpose of this paper is to generalize Kohnen's results to the case of arbitrary odd level and of arbitrary character, and to deduce that, under the assumption of multiplicity one theorem, the square of Fourier coefficients of modular forms of half integral weight, belonging to Kohnen's spaces of arbitrary odd level and of arbitrary character, essentially coincides with the critical values of zeta functions twisted with quadratic character, which refines our previous results [8] and [9]. The method of this paper is basically the same as that of [6].

Section 0 is a preliminary section. In Section 1, we shall recall the definition of Kohnen's space, which is a subspace of modular forms of half integral weight, and Hecke operators acting on there. We then introduce Poincaré series of Kohnen's space and modular forms of integral weight associated with a space of quadratic forms. Furthermore, we determine explicit Fourier coefficients of Fourier expansion of those.

In Section 2, by virtue of the computation of Gauss sum, we shall derive that a sum of Kloosterman sum is expressed as that of genus characters. Using this, we shall deduce that a certain function which is a series whose terms are modular forms associated with a space of quadratic forms coincides with a series whose terms are Poincare series of Kohnen's space, which plays an essential role in our later argument.

In Section 3, using the function introduced in Section 2 as a kernel function, we shall establish a correspondence $\Psi$ from Kohnen's space $S_{k+1 / 2}(N, \chi)$ of modular forms $f(z)=$ $\sum_{n=1,(-1)^{k} n \equiv 0,1(4)}^{\infty} a(n) e[n z]$ of half integral weight $k+1 / 2$ to the space $S_{2 k}\left(N, \chi^{2}\right)$ of modular forms of integral weight $2 k$. Using the identity mentioned above, we shall explicitly determine Fourier coefficients of $F=\Psi(f)$ in terms of those of $f \in S_{k+1 / 2}(N, \chi)$. Moreover, it is shown that $\Psi$ commutes with the action of Hecke operators. Employing the fact that our correspondence $\Psi$ has an integral expression and the identity mentioned above, the Fourier coefficients of $\Psi^{*}(F)$ is expressed by a certain cycle integral of $F$ with the adjoint mapping $\Psi^{*}$ of $\Psi$.

In Section 4, under the assumption that $f$ is an eigenfunction of Hecke operators and $\Psi(f)$ is a new form in $S_{2 k}\left(N, \chi^{2}\right)$, it is verified that $\Psi(f)$ is equal to $c F$ with a Fourier coefficient $c$ of $f$ and the primitive form $F$ attached to $\Psi(f)$. Furthermore, we can show that $\Psi^{*}(F)$ is an eigenfunction of Hecke operators using the properties that $\Psi$ commutes with the action of Hecke operators and those of Hecke operators. By the assumption that $f$ satisfies the multiplicity one theorem of Hecke operators, we see that there is a constant $c^{\prime}$ such that $\Psi^{*}(F)=c^{\prime} f$, which means that $f$ can be recovered from $\Psi(f)$. Using these facts, the property of the adjoint mapping $\Psi^{*}$ of $\Psi$ and results in Section 3, we may derive that the square of Fourier coefficients of $f$ is determined by cycle integrals of $F$. From these, it is deduced that the square of Fourier coefficients $a(|D|)$ of $f$ with a fundamental discriminant $D$ is essentially equal to the critical values of $L$-series of $F$ twisted with quadratic characters. We mention that our results are closely related with those of [17, Theorem 3.6.B], but there are several differences between Shimura's results and ours. There he showed that the critical value of the zeta function attached to $F$ are represented by the Fourier coefficients of 
certain modular forms related with $f$. We determine explicitly $a(|D|)$ in terms of the critical values of the zeta function attached to $F$ with a fundamental discriminant $D$. Recently, using Ueda's results, Sakata [15] showed a relation between Fourier coefficients of modular forms in $S_{k+1 / 2}(N, \chi)$ and the critical value of the zeta function associated with the modular form in $S_{2 k}\left(N, \chi^{2}\right)$ determined by the Shimura correspondence under the condition that $\chi^{2}=1$. We note that Ueda [19] investigated the problem of the multiplicity of Hecke operators acting on $S_{k+1 / 2}(N, \chi)$ with $\chi^{2}=1$.

Finally, the authors are grateful to the referee who suggested revisions of the original version of this paper.

0. Notation and preliminaries. We denote by $\boldsymbol{Z}, \boldsymbol{Q}, \boldsymbol{R}$ and $\boldsymbol{C}$ the ring of rational integers, the rational number field, the real number field and the complex number field, respectively. For a $z \in \boldsymbol{C}$, we define $\sqrt{z}=z^{1 / 2}$ so that $-\pi / 2<\arg z^{1 / 2} \leqq \pi / 2$ and put $z^{k / 2}=(\sqrt{z})^{k}$ for every $k \in \boldsymbol{Z}$. Further we put $e[z]=\exp (2 \pi i z)$ for $z \in \boldsymbol{C}$. For a commutative ring $R$ with identity element, we denote by $S L(2, R)$ the special linear group of all matrices of degree 2 with coefficients in $R$ and $\mathfrak{H}$ the complex upper half plane, i.e.,

$$
S L(2, R)=\left\{\left(\begin{array}{ll}
a & b \\
c & d
\end{array}\right) \mid a, b, c, d \in R \text { and } a d-b c=1\right\}
$$

and

$$
\mathfrak{H}=\{z=x+i y \in \boldsymbol{C} \mid x, y \in \boldsymbol{R} \text { and } y>0\} .
$$

For a positive integer $m$, we put

$$
\Gamma_{0}(m)=\left\{\left(\begin{array}{ll}
a & b \\
c & d
\end{array}\right) \in S L(2, \boldsymbol{Z}) \mid c \equiv 0 \quad(\bmod m)\right\} .
$$

Define an action of $S L(2, \boldsymbol{R})$ on $\mathfrak{H}^{*}=\mathfrak{H} \cup \boldsymbol{R} \cup\{\infty\}$ by

$$
z \in \mathfrak{H}^{*} \mapsto \gamma(z)=\frac{a z+b}{c z+d} \in \mathfrak{H}^{*} \text { for } \gamma=\left(\begin{array}{ll}
a & b \\
c & d
\end{array}\right) \in S L(2, \boldsymbol{R})
$$

and for $z \in \mathfrak{H}^{*}$. The symbol $\left(\frac{*}{*}\right)$ indicates the same as that of [14]. For $c \in \boldsymbol{Z}$, the notation $\sum_{n(c)}\left(\right.$ resp. $\left.\sum_{n(c) \times}\right)$ means that $n$ runs over all $n \in \boldsymbol{Z} / c \boldsymbol{Z}$ (resp. $\left.(\boldsymbol{Z} / c \boldsymbol{Z})^{\times}\right)$.

1. Kohnen's spaces and modular forms. For integers $l, M$ and a Dirichlet character $\psi$ modulo $M$, we denote by $S_{l}(M, \psi)$ the space of modular cusp forms of weight $l$ with level $M$ and character $\psi$. Further we denote by $T_{l, M, \psi}(p)$ the Hecke operators on $S_{l}(M, \psi)$ for every prime $p$. Let $N$ be an odd integer, $\chi$ a Dirichlet character modulo $N$ such that $\chi(-1)=\varepsilon$ and $k$ a non negative integer. We denote by $S_{k+1 / 2}(N, \chi)$ the subspace of $S_{\kappa}(4 N, \tilde{\chi})$ consisting of those $f$ whose Fourier expansion has the form

$$
f(z)=\sum_{\varepsilon(-1)^{k} n \equiv 0,1(4)} a(n) e[n z],
$$


where $\tilde{\chi}=\left(\frac{4 \varepsilon}{*}\right) \chi, \kappa=2 k+1$ and $S_{\kappa}(4 N, \tilde{\chi})$ is the space of cusp forms of half integral weight $k+1 / 2$ with level $4 N$ and a character $\tilde{\chi}$ modulo $4 N$ in the sense of Shimura [16]. If $f$ and $g$ are cusp forms of $S_{\kappa}(4 N, \tilde{\chi})$, we determine the Petersson inner product $\langle f, g\rangle$ by

$$
\langle f, g\rangle=\frac{1}{i_{4 N}} \int_{\Gamma_{0}(4 N) \backslash \mathfrak{H}} f(z) \overline{g(z)} y^{k+1 / 2-2} d x d y,
$$

where $z=x+i y \in \mathfrak{H}$ and $i_{M}=\left[S L(2, \boldsymbol{Z}): \Gamma_{0}(M)\right]$. We denote by Pr the projection from $S_{\kappa}(4 N, \tilde{\chi})$ to $S_{k+1 / 2}(N, \chi)$. For a positive integer $m$ we determine the $m$-th Poincare series $\wp_{k, 4 N, m, \tilde{\chi}}$ by the relation

$$
\left\langle g, \wp_{k, 4 N, m, \tilde{\chi}}\right\rangle=i_{4 N}^{-1} \frac{\Gamma(k-1 / 2)}{(4 \pi m)^{k-1 / 2}} a_{g}(m)
$$

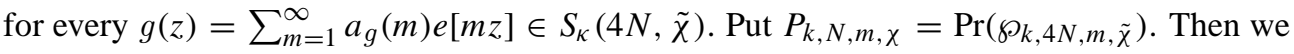
have the following Fourier expansion

$$
P_{k, N, m, \chi}(z)=\sum_{n=1, \varepsilon(-1)^{k} n \equiv 0,1(4)}^{\infty} g_{k, N, m, \chi}(n) e[n z] .
$$

By the same method as that of [6, pp. 251-257], we have the following proposition.

Proposition 1.1. Let $m$ be a positive integer such that $\varepsilon(-1)^{k} m \equiv 0,1(\bmod 4)$. Then

$$
\begin{aligned}
& g_{k, N, m, \chi}(n) \\
& =\frac{2}{3}\left(\delta_{m, n}+(-1)^{[(k+1) / 2]} \sqrt{2} \pi(n / m)^{k / 2-1 / 4} \sum_{c=1, N \mid c}^{\infty} H_{c}(n, m, \chi) J_{k-1 / 2}\left(\frac{\pi}{c} \sqrt{m n}\right)\right)
\end{aligned}
$$

with

$$
\begin{aligned}
& H_{N c^{\prime}}(n, m, \chi)=\left(1-(-1)^{k} i\right)\left(1+\left(\frac{4}{N c^{\prime}}\right)\right) \\
& \times \frac{1}{4 N c^{\prime}} \sum_{\delta\left(4 N c^{\prime}\right)^{\times}}\left(\frac{4 N c^{\prime}}{\delta}\right)\left(\frac{-4}{\delta}\right)^{k+1 / 2} e_{4 N c^{\prime}}\left(n \delta+m \delta^{-1}\right) \bar{\chi}(\delta)\left(\frac{4 \varepsilon}{\delta}\right),
\end{aligned}
$$

where $J_{k-1 / 2}(t)=(t / 2)^{k-1 / 2} \sum_{r=0}^{\infty}(-1)^{r}(t / 2)^{2 r}\{r ! \Gamma(k+r+1 / 2)\}^{-1}$, the sum $\sum_{\delta(c)^{\times}}$(resp. $\left.\sum_{\delta(c)}\right)$ is taken over all $\delta \in(\boldsymbol{Z} / c \boldsymbol{Z})^{\times}$(resp. $\left.\delta \in \boldsymbol{Z} / c \boldsymbol{Z}\right), \delta^{-1}$ means an integer such that $\delta \cdot \delta^{-1} \equiv 1\left(\bmod 4 N c^{\prime}\right)$, and $e_{l}(x)=e[x / l]$.

We review the definition of Hecke operators given in [5, Section 3]. For a prime $p$ $(p \nmid N)$, we define a Hecke operator $T_{k+1 / 2, N, \chi}\left(p^{2}\right)$ by 
(1-6)

$$
\begin{aligned}
& T_{k+1 / 2, N, \chi}\left(p^{2}\right) f(z) \\
& =\sum_{n=1, \varepsilon(-1)^{k} n=0,1(4)}^{\infty}\left\{a\left(p^{2} n\right)+\chi(p)\left(\frac{\varepsilon(-1)^{k} n}{p}\right) p^{k-1} a(n)+\chi\left(p^{2}\right) p^{2 k-1} a\left(n / p^{2}\right)\right\} e[n z]
\end{aligned}
$$

for every $f(z)=\sum_{n=1, \varepsilon(-1)^{k} n \equiv 0,1(4)}^{\infty} a(n) e[n z] \in S_{k+1 / 2}(N, \chi)$. For a prime $p(p \mid N)$, put

$$
U\left(p^{2}\right) f(z)=\sum_{n=1, \varepsilon(-1)^{k} n \equiv 0,1(4)}^{\infty} a\left(p^{2} n\right) e[n z]
$$

for every $f(z)=\sum_{n=1, \varepsilon(-1)^{k} n \equiv 0,1(4)} a(n) e[n z] \in S_{k+1 / 2}(N, \chi)$. Let $N, k$ be positive integers and $\chi$ a Dirichlet character modulo $N$ whose conductor is $N_{1}$. Denote by $\chi_{1}$ the primitive character associated with $\chi$. Put $N_{2}=N / N_{1}$. We consider integers $k, t, D, D^{\prime}$ such that

$(1-8) \quad k \geqq 2,\left(N_{1}, t\right)=1, D, D^{\prime} \equiv 0,1(4),(N, D)=1, \varepsilon(-1)^{k} D>0$ and $D D^{\prime}>0$.

Define a function $f_{k, N_{1}^{2}, t}\left(z ; D, D^{\prime}, \chi_{1}\right)$ on $\mathfrak{H}$ by

$$
f_{k, N_{1}^{2}, t}\left(z ; D, D^{\prime}, \chi_{1}\right)=\sum_{(a, b, c)} \omega_{D}(a, b, c)\left(a z^{2}+b z+c\right)^{-k} \chi_{1}(c),
$$

where $(a, b, c)$ runs over all integers in $Z^{3}$ such that $b^{2}-4 a c=N_{1}^{2} D D^{\prime}$ and $N_{1}^{2} t \mid a$, and $\omega_{D}$ means the symbol given in [6, p. 238]. Then we can easily check the following properties

$$
f_{k, N_{1}^{2}, t}\left(g(z) ; D, D^{\prime}, \chi_{1}\right)=\bar{\chi}_{1}^{2}(\delta)(\gamma z+\delta)^{2 k} f_{k, N_{1}^{2}, t}\left(z ; D, D^{\prime}, \chi_{1}\right)
$$

for every $g=\left(\begin{array}{ll}\alpha & \beta \\ \gamma & \delta\end{array}\right) \in \Gamma_{0}\left(N_{1} t\right)$ and

$$
f_{k, N_{1}^{2}, t}\left(-\bar{z} ; D, D^{\prime}, \chi_{1}\right)=\overline{f_{k, N_{1}^{2}, t}\left(z ; D, D^{\prime}, \bar{\chi}_{1}\right)} .
$$

Next we shall determine explicit Fourier coefficients of $f_{k, N_{1}^{2}, t}\left(z ; D, \varepsilon(-1)^{k} m, \chi_{1}\right)$. This function can be decomposed into

$$
f_{k, N_{1}^{2}, t}\left(z ; D, \varepsilon(-1)^{k} m, \chi_{1}\right)=f_{k, N_{1}^{2}, t}^{0}(z)+2 \sum_{a=1, N_{1}^{2} t \mid a}^{\infty} f_{k, N_{1}^{2}, t}^{a}(z)
$$

with

and

$$
f_{k, N_{1}^{2}, t}^{0}(z)=2 \sum_{b, c \in \mathbf{Z}, b>0, b^{2}=|D| m} \omega_{D}(0, b, c)(b z+c)^{-k} \chi_{1}(c)
$$

$$
\begin{aligned}
f_{k, N_{1}^{2}, t}^{a}(z)= & \sum_{b \in \boldsymbol{Z}, b^{2} \equiv N_{1}^{2}|D| m(4 a)} \omega_{D}\left(a, b, \frac{b^{2}-N_{1}^{2}|D| m}{4 a}\right) \\
& \times\left(a z^{2}+b z+\frac{b^{2}-N_{1}^{2}|D| m}{4 a}\right)^{-k} \chi_{1}\left(\frac{b^{2}-N_{1}^{2}|D| m}{4 a}\right) .
\end{aligned}
$$


We have

$$
\begin{aligned}
& f_{k, N_{1}^{2}, t}^{N_{1}^{2} t a^{\prime}}(z)=\sum_{b^{\prime}\left(2 N_{1} t a^{\prime}\right),\left(b^{\prime}\right)^{2} \equiv|D| m\left(4 t a^{\prime}\right)} \omega_{D}\left(N_{1}^{2} t a^{\prime}, N_{1} b^{\prime}, \frac{\left(b^{\prime}\right)^{2}-|D| m}{4 a^{\prime} t}\right) \\
& \times \chi_{1}\left(\frac{\left(b^{\prime}\right)^{2}-|D| m}{4 a^{\prime} t}\right) \sum_{n \in \boldsymbol{Z}}\left(N_{1}^{2} t a^{\prime}(z+n)^{2}+N_{1} b^{\prime}(z+n)+\frac{\left(N_{1} b^{\prime}\right)^{2}-N_{1}^{2}|D| m}{4 N_{1}^{2} t a^{\prime}}\right)^{-k} .
\end{aligned}
$$

Here we need a formula for $c>0$

$$
\begin{aligned}
& \int_{-\infty+i C}^{\infty+i C}\left(a z^{2}+b z+\frac{b^{2}-N_{1}^{2}|D| m}{4 a}\right)^{-k} e^{-2 \pi i n z} d z \\
& =\frac{(-1)^{k} 2^{k+1 / 2} \pi^{k+1} n^{k-1 / 2}}{\left(N_{1}^{2}|D| m\right)^{k / 2-1 / 4} \sqrt{a}(k-1) !} J_{k-1 / 2}\left(\frac{\pi n \sqrt{N_{1}^{2}|D| m}}{a}\right) e^{\pi i n b / a}
\end{aligned}
$$

(cf. [1, 29.3.57]). Hence we may derive the following.

$$
\begin{aligned}
f_{k, N_{1}^{2}, t}^{N_{1}^{2} t a^{\prime}}(z)= & \frac{(-1)^{k} 2^{k+1 / 2} \pi^{k+1}}{N_{1}^{k+1 / 2} \sqrt{t}(|D| m)^{k / 2-1 / 4} \sqrt{a^{\prime}}(k-1) !} \\
& \times \sum_{n=1}^{\infty} n^{k-1 / 2} S_{N_{1} t a^{\prime}, D, \varepsilon(-1)^{k} m, \chi_{1}}(|D| m, n) J_{k-1 / 2}\left(\frac{\pi n \sqrt{|D| m}}{N_{1} t a^{\prime}}\right) e^{2 \pi i n z}
\end{aligned}
$$

with

$$
\begin{aligned}
& S_{N_{1} a, D, \varepsilon(-1)^{k} m, \chi_{1}}(|D| m, n) \\
& \quad=\sum_{b\left(2 N_{1} a\right), b^{2} \equiv|D| m(4 a)} \omega_{D}\left(N_{1}^{2} a, N_{1} b, \frac{b^{2}-|D| m}{4 a}\right) \chi_{1}\left(\frac{b^{2}-|D| m}{4 a}\right) e_{2 N a}(n b) .
\end{aligned}
$$

Next we shall compute $f_{k, N_{1}^{2}, t}^{0}(z)$. If $f=\sqrt{m /|D|} \in \boldsymbol{Z}$, then we may check

$$
\begin{aligned}
f_{k, N_{1}^{2}, t}^{0}(z) & =2 \sum_{c \in \boldsymbol{Z}}\left(\frac{D}{c}\right)\left(N_{1}|D| f z+c\right)^{-k} \chi_{1}(c) \\
& =2 \sum_{r\left(D N_{1}\right)}\left(\frac{D}{r}\right) \chi_{1}(r)\left(N_{1}|D|\right)^{-k} \sum_{n \in \boldsymbol{Z}}\left(f z+\frac{r}{|D| N_{1}}+n\right)^{-k} .
\end{aligned}
$$

Using Lipschitz's formula

$$
\sum_{n \in \boldsymbol{Z}}(\tau+n)^{-s}=\frac{(2 \pi)^{s} e^{-\pi i s / 2}}{\Gamma(s)} \sum_{n=1}^{\infty} n^{s-1} e^{2 \pi i n \tau} \quad(\tau \in \mathfrak{H}, \Re(s)>1)
$$


we may verify that

$$
\begin{aligned}
& f_{k, N_{1}^{2}, t}^{0}(z)=\frac{2\left(N_{1}|D|\right)^{-k}(-i)^{k}(2 \pi)^{k}}{(k-1) !}\left(\frac{D}{N_{1}}\right) \chi_{1}(|D|) W\left(\chi_{1}\right) \\
& \quad \times\left(\frac{D}{-1}\right)^{1 / 2}|D|^{1 / 2} \begin{cases}\sum_{n=1}^{\infty} \bar{\chi}_{1}(n)\left(\frac{D}{n}\right) n^{k-1} e^{2 \pi i n f z} & \text { if } f=\sqrt{m /|D|} \in \boldsymbol{Z}, \\
0 & \text { otherwise, }\end{cases}
\end{aligned}
$$

where $W\left(\chi_{1}\right)$ is the Gauss sum of $\chi_{1}$. We may deduce the following

Proposition 1.2. Put

$$
f_{k, N_{1}^{2}, t}\left(z ; D, \varepsilon(-1)^{k} m, \chi_{1}\right)=\sum_{n=1}^{\infty} c_{k, N_{1}^{2}, t}\left(n ; D, \varepsilon(-1)^{k} m\right) e[n z] .
$$

Then

$$
\begin{aligned}
c_{k, N_{1}^{2}, t}\left(n ; D, \varepsilon(-1)^{k} m\right) \\
=2 \frac{(-1)^{k} 2^{k+1 / 2} \pi^{k+1}}{N_{1}^{k+1 / 2}(|D| m)^{k / 2-1 / 4}(k-1) !} \sum_{a^{\prime}=1}^{\infty} n^{k-1 / 2} \sqrt{t a^{\prime}} S_{N_{1} t a^{\prime}, D, \varepsilon(-1)^{k} m, \chi_{1}}(|D| m, n) \\
\\
\times J_{k-1 / 2}\left(\frac{\pi n \sqrt{|D| m}}{N_{1} t a^{\prime}}\right)+2 \frac{\left(N_{1}|D|\right)^{-k}(-i)^{k}(2 \pi)^{k}}{(k-1) !}\left(\frac{D}{N_{1}}\right) \chi_{1}(|D|) W\left(\chi_{1}\right)\left(\frac{D}{-1}\right)^{1 / 2} \\
\quad \times|D|^{1 / 2} \begin{cases}\bar{\chi}_{1}(n / f)\left(\frac{D}{n / f}\right)(n / f)^{k-1} & \text { if } f=\sqrt{m /|D|} \in \boldsymbol{Z} \text { and } n / f \in \boldsymbol{Z}, \\
0 & \text { otherwise. }\end{cases}
\end{aligned}
$$

2. Gauss sum, Kloosterman sum and a basic identity. Let $N$ be an odd integer, and $\chi$ a Dirichlet character modulo $N$ whose conductor is $N_{1}$. Put $\varepsilon=\chi(-1)$ and $N_{2}=N / N_{1}$. We denote by $\chi_{1}$ the primitive character associated with $\chi$. For $c \in Z(>0)\left(N_{1} \mid c\right), n$ and $h \in \boldsymbol{Z}$, put

(2-1) $\mathfrak{F}_{4 c, \chi_{1}}(Q(n))=\frac{1}{4 c}\left(1+\left(\frac{4}{c}\right)\right) \sum_{n(2 c), \delta(4 c)}\left(\frac{4 c}{\delta}\right) \chi_{1}(\delta)\left(1-\left(\frac{-4}{\delta}\right) i\right) e_{4 c}(\delta Q(n))$,

where $Q(n)=D n^{2}-2 h n+\varepsilon(-1)^{k} m$ and $D, m$ are the integers given in (1-8). Further put $c=c_{0}^{\prime} c_{0}$, where $c_{0}$ is odd and $c_{0}^{\prime}$ is a power of 2 . Then the quadratic reciprocity law implies that

$$
\mathfrak{F}_{4 c, \chi_{1}}(Q(n))=\chi_{1}\left(4 c_{0}^{\prime}\right) \mathfrak{F}_{4 c_{0}^{\prime}}(Q(n)) \mathfrak{F}_{c_{0}, \chi_{1}}(Q(n))
$$

with

$$
\mathfrak{F}_{4 c_{0}^{\prime}}(Q(n))=\frac{1}{4 c_{0}^{\prime}}\left(1+\left(\frac{4}{c_{0}^{\prime}}\right)\right) \sum_{n\left(2 c_{0}^{\prime}\right), \delta\left(4 c_{0}^{\prime}\right)}\left(\frac{4 c_{0}^{\prime}}{\delta}\right)\left(1-\left(\frac{-4}{\delta}\right) i\right) e_{4 c_{0}^{\prime}}(\delta Q(n))
$$


and

$$
\mathfrak{F}_{c_{0}, \chi_{1}}(Q(n))=\frac{1}{c_{0}}\left(\frac{-4}{c_{0}}\right)^{-1 / 2} \sum_{n, \delta\left(c_{0}\right)}\left(\frac{\delta}{c_{0}}\right) \chi_{1}(\delta) e_{c_{0}}(\delta Q(n)) .
$$

Put

$$
N_{1}=\prod_{i=1}^{l} p_{i}^{v_{i}}, \quad c_{0}=\prod_{i=1}^{l} p_{i}^{\lambda_{i}+v_{i}} \prod_{i=l+1}^{s} p_{i}^{\lambda_{i}}, \quad c_{0}^{\prime}=2^{\lambda},
$$

where $p_{i}(1 \leqq i \leqq s)$ are prime and $p_{i} \neq p_{j}(i \neq j)$. According as the natural isomorphism of group $\left(\boldsymbol{Z} / N_{1} Z\right)^{\times} \cong \prod_{i=1}^{l}\left(\boldsymbol{Z} / p_{i}^{v_{i}} \boldsymbol{Z}\right)^{\times}$, we can decompose $\chi_{1}$ into $\prod_{i=1}^{l} \chi_{1, i}$. Using this, we may derive

$$
\begin{aligned}
& \mathfrak{F}_{4 c, \chi_{1}}(Q(n))=\chi_{1}\left(2^{\lambda+2} \prod_{i=l+1}^{s} p_{i}^{\lambda_{i}}\right) \\
& \quad \times \prod_{i=1}^{l} \chi_{1, i}\left(\frac{\prod_{j=1}^{l} p_{j}^{\lambda_{j}+v_{j}}}{p_{i}^{\lambda_{i}+v_{i}}}\right) \mathfrak{F}_{2^{\lambda+2}}(Q(n)) \prod_{i=1}^{l} \mathfrak{F}_{p_{i}^{\lambda_{i}+v_{i}}, \chi_{1, i}}(Q(n)) \prod_{i=l+1}^{s} \mathfrak{F}_{p_{i}^{\lambda_{i}}}(Q(n)) .
\end{aligned}
$$

For positive integers $a, h$ and $m$, define $C_{D, m}\left(N_{1} a, h, \chi_{1}\right)$ by

$$
\begin{aligned}
C_{D, m}\left(N_{1} a, h, \chi_{1}\right)= & \left(4 N_{1} a\right)^{-1} \sum_{n\left(2 N_{1} a\right)} e_{2 N_{1} a}(-h n) \\
& \times \sum_{d \mid\left(N_{1} a, n\right)}\left(\frac{D}{d}\right)\left(4 N_{1} a / d\right)^{1 / 2} \bar{\chi}_{1}(d) H_{N_{1} a / d}\left(m, n^{2}|D| d^{2}, \chi_{1}\right) .
\end{aligned}
$$

Put $a=2^{e} \prod_{i=1}^{s} p_{i}^{\alpha_{i}}=2^{e} \tilde{a_{1}} \tilde{a_{2}}$, where $\left(\tilde{a_{1}}, \tilde{a_{2}}\right)=1,\left(N_{1}, \tilde{a_{2}}\right)=1$. Then, by (2.3), we may deduce that

$$
\begin{aligned}
& C_{D, m}\left(N_{1} a, h, \chi_{1}\right) \\
&=\left(\frac{\varepsilon}{-1}\right)^{1 / 2}\left(4 N_{1} a\right)^{-1} \sum_{d \mid N_{1} a}\left(\frac{D}{d}\right)\left(4 N_{1} a / d\right)^{1 / 2} \bar{\chi}_{1}(d) \mathfrak{F}_{4 N_{1} a / d, \bar{\chi}_{1}}(Q(n)) \\
&=\left(\frac{\varepsilon}{-1}\right)^{1 / 2}\left(\frac{1}{2^{e+2}} \sum_{d \mid 2^{e}}\left(\frac{D}{d}\right)\left(2^{e+2} / d\right)^{1 / 2} \bar{\chi}_{1}(d) \bar{\chi}_{1}\left(2^{e+2} / d\right) \mathfrak{F}_{2^{e+2} / d}(Q(n))\right) \\
& \times \prod_{i=1}^{l}\left(\left(p_{i}^{v_{i}+\alpha_{i}}\right)^{-1 / 2} \bar{\chi}_{1, i}\left(\frac{\prod_{j=1}^{l} p_{j}^{\alpha_{j}+v_{j}}}{p_{i}^{\alpha_{i}+v_{i}}}\right) \mathfrak{F}_{p_{i}^{\alpha_{i}+v_{i}}, \bar{\chi}_{1, i}}(Q(n))\right) \\
& \times \prod_{i=l+1}^{s}\left(\frac{1}{p_{i}^{\alpha_{i}}} \sum_{d \mid p_{i}^{\alpha_{i}}}\left(\frac{D}{d}\right)\left(p_{i}^{\alpha_{i}} / d\right)^{1 / 2} \bar{\chi}_{1}(d) \bar{\chi}_{1}\left(p_{i}^{\alpha_{i}} / d\right) \mathfrak{F}_{p_{i}^{\alpha_{i}} / d}(Q(n))\right) .
\end{aligned}
$$


For $i(1 \leqq i \leqq l)$, we now calculate $\mathfrak{F}_{p_{i}^{\alpha_{i}+v_{i}, \bar{\chi}_{1, i}}}(Q(n))$.

$$
\begin{aligned}
\mathfrak{F}_{p_{i}^{\alpha_{i}+v_{i}}, \bar{\chi}_{1, i}}(Q(n))= & \frac{1}{p_{i}^{\alpha_{i}+v_{i}}}\left(\frac{-4}{p_{i}^{\alpha_{i}+v_{i}}}\right)^{-1 / 2} \\
& \times \sum_{\delta\left(p_{i}^{\alpha_{i}+v_{i}}\right)^{\times}}\left(\frac{\delta}{p_{i}^{\alpha_{i}+v_{i}}}\right) \sum_{n\left(p_{i}^{\alpha_{i}+v_{i}}\right)} \bar{\chi}_{1, i}(\delta) e_{p_{i}^{\alpha_{i}+v_{i}}}(\delta Q(n)) .
\end{aligned}
$$

We can put $\delta=\alpha+\beta p_{i}^{v_{i}}$ with $\alpha \in\left(\boldsymbol{Z} / p_{i}^{\alpha_{i}+v_{i}} \boldsymbol{Z}\right)^{\times}$and $\beta \in \boldsymbol{Z} / p_{i}^{\alpha_{i}} \boldsymbol{Z}$. Assume that $p_{i} \nmid D$. Determine $D^{-1} \in \boldsymbol{Z}$ such that $Q(n) \equiv D^{-1}\left((D n-h)^{2}-\Delta\right)\left(\bmod p_{i}^{\alpha_{i}+v_{i}}\right)$, where $\Delta=$ $h^{2}-\varepsilon(-1)^{k} D m$. Then we have

$$
\sum_{n\left(p_{i}^{\alpha_{i}+v_{i}}\right)} e_{p_{i}^{\alpha_{i}+v_{i}}}(\delta Q(n))=e_{p_{i}^{\alpha_{i}+v_{i}}}\left(-\delta D^{-1} \Delta\right)\left(\frac{\delta D}{p_{i}^{\alpha_{i}+v_{i}}}\right)\left(\frac{-4}{p_{i}^{\alpha_{i}+v_{i}}}\right)^{1 / 2} p_{i}^{\left(\alpha_{i}+v_{i}\right) / 2} .
$$

Therefore we may confirm

$$
\begin{aligned}
\mathfrak{F}_{p_{i}^{\alpha_{i}+v_{i}}, \bar{\chi}_{1, i}}(Q(n))= & \frac{1}{p_{i}^{\left(\alpha_{i}+v_{i}\right) / 2}}\left(\frac{D}{p_{i}^{\alpha_{i}+v_{i}}}\right) \\
& \times \sum_{\alpha\left(p_{i}^{v_{i}}\right) \times} \sum_{\beta\left(p_{i}^{\alpha_{i}}\right)} \bar{\chi}_{1, i}(\alpha) e_{p_{i}^{\alpha_{i}+v_{i}}}\left(-\alpha D^{-1} \Delta\right) e_{p_{i}^{\alpha_{i}}}\left(-\beta D^{-1} \Delta\right),
\end{aligned}
$$

which is zero unless $p_{i}^{\alpha_{i}} \mid D^{-1} \Delta$. Under this condition, we may check

$$
\sum_{\alpha\left(p_{i}^{v_{i}}\right) \times} \bar{\chi}_{1, i}(\alpha) e_{p_{i}^{\alpha_{i}+v_{i}}}\left(-\alpha D^{-1} \Delta\right)=W\left(\bar{\chi}_{1, i}\right) \chi_{1, i}\left(-D^{-1}\right) \chi_{1, i}\left(\Delta / p_{i}^{\alpha_{i}}\right) .
$$

It follows from this that

$$
\begin{aligned}
& \prod_{i=1}^{l}\left(\frac{1}{\left(p_{i}^{v_{i}+\alpha_{i}}\right)^{1 / 2}} \bar{\chi}_{1, i}\left(\prod_{j=1}^{l} p_{j}^{\alpha_{j}+v_{j}} / p_{i}^{\alpha_{i}+v_{i}}\right)\right) \mathfrak{F}_{p_{i}^{\alpha_{i}+v_{i}}, \bar{\chi}_{1, i}}(Q(n)) \\
& \quad=\prod_{i=1}^{l} \frac{1}{p_{i}^{v_{i}}} W\left(\bar{\chi}_{1, i}\right) \chi_{1, i}\left(-D^{-1}\right) \bar{\chi}_{1, i}\left(\frac{\prod_{j=1}^{l} p_{j}^{v_{j}}}{p_{i}^{v_{i}}}\right) \chi_{1, i}\left(\frac{\Delta}{\prod_{j=1}^{l} p_{j}^{\alpha_{j}}}\right)\left(\frac{D}{p_{i}^{\alpha_{i}+v_{i}}}\right) .
\end{aligned}
$$

Since $W\left(\bar{\chi}_{1}\right)=\prod_{i=1}^{l} W\left(\bar{\chi}_{1, i}\right) \bar{\chi}_{1, i}\left(N_{1} / p_{i}^{\nu_{i}}\right),(2-10)$ is equal to

$$
\begin{cases}\frac{1}{N_{1}} W\left(\bar{\chi}_{1}\right) \chi_{1}\left(-D^{-1}\right) \chi_{1}\left(\Delta / \tilde{a_{1}}\right)\left(\frac{D}{N_{1}}\right)\left(\frac{D}{\tilde{a_{1}}}\right) & \text { if } \tilde{a_{1}} \mid \Delta \\ 0 & \text { otherwise }\end{cases}
$$


Assume that $i \geqq l+1$ and $p_{i} \nmid D$. Then we have

$$
\begin{gathered}
\frac{1}{p_{i}^{\alpha_{i}}} \sum_{d \mid p_{i}^{\alpha_{i}}}\left(\frac{D}{d}\right)\left(p_{i}^{\alpha_{i}} / d\right)^{1 / 2} \bar{\chi}_{1, i}(d) \bar{\chi}_{1, i}\left(p_{i}^{\alpha_{i}} / d\right) \mathfrak{F}_{p_{i}^{\alpha_{i}} / d, \bar{\chi}_{1}}(Q(n)) \\
=\frac{\bar{\chi}_{1}\left(p_{i}^{\alpha_{i}}\right)}{p_{i}^{\alpha_{i}}}\left(\frac{D}{p_{i}^{\alpha_{i}}}\right) \sum_{l=0}^{\alpha_{i}}\left(\frac{D}{p_{i}^{l}}\right)\left(p_{i}^{l}\right)^{1 / 2} \mathfrak{F}_{p_{i}^{l}}(Q(n)) .
\end{gathered}
$$

By virtue of [6, pp. 259-264], (2-12) is equal to

$$
\begin{cases}\bar{\chi}_{1}\left(p_{i}^{\alpha_{i}}\right)\left(\frac{D}{p_{i}^{\alpha_{i}}}\right) & \text { if } p_{i}^{\alpha_{i}} \mid \Delta, \\ 0 & \text { otherwise. }\end{cases}
$$

When $i \geqq l+1, \quad p_{i} \mid D$ and $p_{i}$ is odd, we may verify that

$$
\begin{aligned}
& \frac{1}{p_{i}^{\alpha_{i}}} \sum_{d \mid p_{i}^{\alpha_{i}}}\left(\frac{D}{d}\right)\left(p_{i}^{\alpha_{i}} / d\right)^{1 / 2} \bar{\chi}_{1}(d) \mathfrak{F}_{p_{i}^{\alpha_{i}} / d, \bar{\chi}_{1}}(Q(n)) \\
& \quad= \begin{cases}\bar{\chi}_{1}\left(p_{i}^{\alpha_{i}}\right)\left(\frac{p_{i}^{*}}{\Delta / p_{i}^{\alpha_{i}}}\right)\left(\frac{D / p_{i}^{*}}{p_{i}^{\alpha_{i}}}\right) & \text { if } p_{i}^{\alpha_{i}}\left|\Delta, p_{i}\right| D \text { and } p_{i} \text { is odd, } \\
0 & \text { otherwise, }\end{cases}
\end{aligned}
$$

where $p_{i}^{*}$ is the symbol given in [6, pp. 262-264]. Applying the above argument due to [6, pp. 259-264], we may find that

$$
\begin{aligned}
C_{D, m}\left(N_{1} a, h, \chi_{1}\right)=\left(\frac{\varepsilon}{-1}\right)^{1 / 2} N_{1}^{-1} W\left(\bar{\chi}_{1}\right) \chi_{1}\left(-D^{-1}\right)\left(\frac{D}{N_{1}}\right) \\
\times \chi_{1}(\Delta / 4 a) \begin{cases}\prod_{p^{v} \| 4 a}\left(\frac{D / p^{*}}{p^{v}}\right)\left(\frac{p^{*}}{\left(h^{2}-|D| m\right) / p^{v}}\right) & \text { if } h^{2} \equiv|D| m \quad(\bmod 4 a), \\
0 & \text { otherwise, }\end{cases}
\end{aligned}
$$

where $p$ is a prime and $\alpha \| \beta$ means $\alpha, \beta \in \boldsymbol{Z}, \alpha \mid \beta$ and $(\alpha, \beta / \alpha)=1$. We put

$$
\tilde{G}\left(\bar{\chi}_{1}, D\right)=W\left(\bar{\chi}_{1}\right) \chi_{1}\left(-D^{-1}\right)\left(\frac{D}{N_{1}}\right) \text {. }
$$

By virtue of (2-15), we may confirm that

$$
\begin{aligned}
& \left(\frac{\varepsilon}{-1}\right)^{1 / 2} \tilde{G}\left(\bar{\chi}_{1}, D\right) N_{1}^{-1} S_{N_{1} a, D, \varepsilon(-1)^{k} m, \chi_{1}}(|D| m, n) \\
& =\sum_{d \mid(a, n)}\left(\frac{D}{d}\right)\left(N_{1} a / d\right)^{1 / 2} \bar{\chi}_{1}(d) H_{N_{1} a / d}\left(m, n^{2}|D| / d^{2}, \chi_{1}\right)
\end{aligned}
$$

for every positive integer $a$. By (2-16), we may derive the following proposition. 
Proposition 2.1. Let $m, n$ be positive integers and $D$ a positive integer such that $D \equiv 0,1(\bmod 4),(N, D)=1$ and $\varepsilon(-1)^{k} D>0$. Then

$$
\begin{aligned}
& \left(\frac{\varepsilon}{-1}\right)^{1 / 2} \tilde{G}\left(\bar{\chi}_{1}, D\right) N_{1}^{-1} \sum_{t\left|N_{2}, t\right| n} \mu(t)\left(\frac{D}{t}\right) \bar{\chi}_{1}(t) S_{N_{1}\left(N_{2} / t\right) a, D, \varepsilon(-1)^{k} m, \chi_{1}}\left(|D| m, \frac{n}{t}\right) \\
& =\sum_{l \mid(a, n),\left(l, N_{2}\right)=1}\left(\frac{D}{l}\right)(N a / l)^{1 / 2} \bar{\chi}_{1}(l) H_{N a / l}\left(m, n^{2}|D| / l^{2}, \chi_{1}\right) \\
& =\sum_{l \mid(a, n)}\left(\frac{D}{l}\right)(N a / l)^{1 / 2} \bar{\chi}(l) H_{N a / l}\left(m, n^{2}|D| / l^{2}, \chi\right) .
\end{aligned}
$$

We assume that $k \geqq 2$ and $D$ is a fundamental discriminant satisfying $\varepsilon(-1)^{k} D>0$ and $(D, N)=1$. Define a function $\Omega_{k, N}(z, \tau ; D, \chi)$ on $\mathfrak{H} \times \mathfrak{H}$ by

$$
\begin{aligned}
& \Omega_{k, N}(z, \tau ; D, \chi)=i_{N} c_{k, D}^{-1} \sum_{m=1, \varepsilon(-1)^{k} m \equiv 0,1(4)}^{\infty} m^{k-1 / 2} \\
& \quad \times\left(\sum_{t \mid N_{2}} \mu(t)\left(\frac{D}{t}\right) \bar{\chi}_{1}(t) t^{k-1} f_{k, N_{1}^{2}, N_{2} / t}\left(t z ; D, \varepsilon(-1)^{k} m, \chi_{1}\right)\right) e[m \tau]
\end{aligned}
$$

for every $z$ and $\tau \in \mathfrak{H}$, where

$$
c_{k, D}=(-1)^{[k / 2]}|D|^{-k+1 / 2} \pi\left(\begin{array}{c}
2 k-2 \\
k-1
\end{array}\right) 2^{-3 k+2}
$$

Then we may deduce the following theorem.

THEOREM 2.2 (A basic identity). Suppose that $k \geqq 2$. Then

$$
\Omega_{k, N}(z, \tau ; D, \chi)=C \sum_{n=1}^{\infty} n^{k-1}\left(\sum_{d \mid n}\left(\frac{D}{d}\right)(n / d)^{k} \bar{\chi}(d) P_{k, N, n^{2}|D| / d^{2}, \chi}(\tau)\right) e[n z]
$$

with

$$
C=i_{N} c_{k, D}^{-1} \frac{3(-1)^{[k / 2]}(2 \pi)^{k}}{(k-1) !}\left(\frac{D}{N_{1}}\right) \chi_{1}(-D) W\left(\chi_{1}\right) \varepsilon^{1 / 2} N_{1}^{-k} .
$$

Proof 1. By Propositions 1.1 and 1.2, comparing the Fourier coefficients of right and left hand sides at $e[m \tau], e[n z]$, we need to verify that 
$(2-20)$

$$
\begin{aligned}
i_{N} c_{k, D}^{-1} m^{k-1 / 2} \sum_{t \mid N_{2}} \mu(t)\left(\frac{D}{t}\right) \bar{\chi}_{1}(t) t^{k-1}\left[\delta(\sqrt{m /|D|}) 2 s(\varepsilon, k)\left(N_{1}|D|\right)^{-k}\right. \\
\quad \times(-1)^{k+[(k+1) / 2]}(2 \pi)^{k}|D|^{1 / 2}(k-1) !^{-1}\left(\frac{D}{N_{1}}\right) \chi_{1}(|D|) \bar{\chi}_{1}\left(\frac{n}{t} \sqrt{|D| / m}\right) W\left(\chi_{1}\right) \\
\quad \times\left(\frac{D}{(n / t) \sqrt{|D| / m}}\right)\left(\frac{n}{t} \sqrt{|D| / m}\right)^{k-1} \delta\left(\frac{n}{t} \sqrt{|D| / m}\right) \\
\quad+2(-1)^{k} 2^{k} \sqrt{2} \pi^{k} \pi\left(N_{1}^{k+1 / 2} \sqrt{N_{2} / t}(|D| m)^{k / 2-1 / 4}(k-1) !\right)^{-1} \\
\left.\quad \times \sum_{a^{\prime}=1}^{\infty} S_{N_{1}\left(N_{2} / t\right) a^{\prime}, D, \varepsilon(-1)^{k} m, \chi_{1}}\left(|D| m, \frac{n}{t}\right){\sqrt{a^{\prime}}}^{-1}(n / t)^{k-1 / 2} J_{k-1 / 2}\left(\frac{\pi n \sqrt{|D| m}}{N a^{\prime}}\right)\right] \\
=\frac{2}{3} C n^{k-1} \sum_{d \mid n}\left(\frac{D}{d}\right)(n / d)^{k} \bar{\chi}(d)\left(\delta_{n^{2}|D| / d^{2}, m}+(-1)^{[(k+1) / 2]} \sqrt{2} \pi\left(\frac{m d^{2}}{n^{2}|D|}\right)^{k / 2-1 / 4}\right. \\
\left.\quad \times \sum_{c=1}^{\infty} H_{N c}\left(m, \frac{n^{2}|D|}{d^{2}}, \chi\right) J_{k-1 / 2}\left(\frac{\pi}{N c} \sqrt{\frac{m n^{2}|D|}{d^{2}}}\right)\right),
\end{aligned}
$$

where

$$
\delta(x)=\left\{\begin{array}{ll}
1 & \text { if } x \in Z, \\
0 & \text { otherwise, }
\end{array} \quad \text { and } \quad s(\varepsilon, k)= \begin{cases}1 & \text { if } \varepsilon=1, \\
\varepsilon^{1 / 2}(-1)^{k} & \text { otherwise }\end{cases}\right.
$$

By virtue of Proposition 2.1, the equalities (2-20) coincides with

$$
\begin{aligned}
C^{\prime} \sum_{t\left|N_{2}, t\right| n} \mu(t)\left[\left(\frac{D}{n \sqrt{|D| / m}}\right) \bar{\chi}_{1}(n \sqrt{|D| / m}) \delta\left(\frac{n}{t} \sqrt{|D| / m}\right) \delta(\sqrt{m /|D|})\right. \\
\quad+(-1)^{[(k+1) / 2]} m^{-1 / 4} \sqrt{2} \pi n^{1 / 2} \sqrt{N}^{-1}\left(\frac{D}{N_{1}}\right) \bar{\chi}_{1}(|D|) W\left(\bar{\chi}_{1}\right) \varepsilon s(\varepsilon, k)^{-1} N_{1}^{-1} \\
\left.\quad \times|D|^{1 / 4} \sum_{a=1}^{\infty} \bar{\chi}_{1}(t)\left(\frac{D}{t}\right) S_{(N / t) a, D, \varepsilon(-1)^{k} m, \chi_{1}}\left(|D| m, \frac{n}{t}\right) \frac{1}{\sqrt{a}} J_{k-1 / 2}\left(\frac{\pi n \sqrt{|D| m}}{N a}\right)\right] \\
=C^{\prime}\left(\sum_{t\left|N_{2}, t\right| n} \mu(t) \delta\left(\frac{n}{t} \sqrt{|D| / m}\right)\left(\frac{D}{n \sqrt{|D| / m}}\right) \bar{\chi}_{1}(n \sqrt{|D| / m}) \delta(\sqrt{m /|D|})\right. \\
\quad+(-1)^{[(k+1) / 2]} m^{-1 / 4} \sqrt{2} \pi|D|^{1 / 4} n^{1 / 2} \sum_{a=1}^{\infty} \sum_{d \mid(a, n)}\left(\frac{D}{d}\right) d^{-1 / 2} \bar{\chi}(d) \\
\left.\quad \times H_{N a / d}\left(m, \frac{n^{2}|D|}{d^{2}}, \chi_{1}\right) J_{k-1 / 2}\left(\frac{\pi n \sqrt{|D| m}}{N a}\right)\right)
\end{aligned}
$$




$$
\begin{aligned}
= & \frac{2}{3} C n^{k-1} \sqrt{m /|D|}^{k}\left[\sum _ { d | n } ( \frac { D } { d } ) ( n / d ) ^ { k } \overline { \chi } ( d ) \left(\sqrt{|D| / m}^{k} \delta_{n^{2}|D| / d^{2}, m}+(-1)^{[(k+1) / 2]}\right.\right. \\
& \left.\left.\times \pi \sqrt{2}\left(\frac{m d^{2}}{n^{2}|D|}\right)^{k / 2-1 / 4} \sqrt{|D| / m}^{k} \sum_{c=1}^{\infty} H_{N c}\left(m, \frac{n^{2}|D|}{d^{2}}, \chi\right) J_{k-1 / 2}\left(\frac{\pi n}{N c d} \sqrt{m|D|}\right)\right)\right] \\
= & \frac{2}{3} C n^{k-1} \sqrt{m /|D|}^{k}\left[\left(\frac{D}{n \sqrt{|D| / m}}\right) \bar{\chi}(n \sqrt{|D| / m}) \delta(n \sqrt{|D| / m})+m^{-1 / 4}(-1)^{[(k+1) / 2]}\right. \\
& \times \pi \sqrt{2}^{1 / 2}|D|^{1 / 4} \sum_{a=1}^{\infty} \sum_{d \mid(n, a)}\left(\frac{D}{d}\right) d^{-1 / 2} \bar{\chi}(d) H_{N a / d}\left(m, \frac{n^{2}|D|}{d^{2}}, \chi\right) \\
& \left.\times J_{k-1 / 2}\left(\frac{\pi n \sqrt{m|D|}}{N a}\right)\right]
\end{aligned}
$$

with

$$
\begin{aligned}
C^{\prime}= & 2 i_{N} c_{k, D}^{-1} m^{k-1 / 2} N_{1}^{-k}|D|^{-k+1 / 2}(2 \pi)^{k} \\
& \times\left(\frac{D}{N_{1}}\right)(-1)^{k+[(k+1) / 2]} \chi_{1}(|D|) W\left(\chi_{1}\right) s(\varepsilon, k) \frac{1}{(k-1) !}\left(n \sqrt{|D| / m}^{k-1} .\right.
\end{aligned}
$$

We can check that

$$
\begin{gathered}
\sum_{t \mid\left(N_{2}, n\right)} \mu(t) \delta\left(\frac{n}{t} \sqrt{|D| / m}\right) \delta(\sqrt{m /|D|})\left(\frac{D}{n \sqrt{|D| / m}}\right) \bar{\chi}_{1}(n \sqrt{|D| / m}) \\
=\sum_{t \mid\left(N_{2}, n\right)} \mu(t) \delta\left(\frac{n}{t f}\right) \delta(\sqrt{m /|D|})\left(\frac{D}{n / f}\right) \bar{\chi}_{1}(n / f) \\
=\delta\left(\sqrt{m /|D|)}\left(\frac{D}{n / f}\right) \bar{\chi}_{1}(n / f) \sum_{t \mid\left(N_{2}, n / f\right)} \mu(t)\right. \\
=\left(\frac{D}{n / f}\right) \bar{\chi}_{1}(n / f) \begin{cases}1 & \text { if }\left(N_{2}, n / f\right)=1, \\
0 & \text { otherwise }\end{cases}
\end{gathered}
$$

with $f=\sqrt{m /|D|}$. Observe that $C^{\prime}=(2 / 3) C n^{k-1} \sqrt{m /|D|}^{k}$. This completes the proof.

3. Shimura-Shintani correspondence and kernel functions. This section is devoted to establishing Shimura correspondences $\Psi$ from $S_{k+1 / 2}(N, \chi)$ to $S_{2 k}\left(N, \chi^{2}\right)$ using kernel functions, and to detemining explicit Fourier coefficients of $\Psi(f)$ in terms of those of $f$ for every $f$ belonging to Kohnen's space $S_{k+1 / 2}(N, \chi)$. Let $N$ be an odd integer and $\chi$ a Dirichlet character. Assume that $D$ is a fundamental discriminant such that $\varepsilon(-1)^{k} D>0$ and $(N, D)=1$. Define

$$
\Psi_{N, \chi, D}(f)(z)=\sum_{n=1}^{\infty}\left(\sum_{d \mid n}\left(\frac{D}{d}\right) \chi(d) d^{k-1} a\left(n^{2}|D| / d^{2}\right)\right) e[n z]
$$


for every $f(\tau)=\sum_{\varepsilon(-1)^{k} n \equiv 0,1(4)} a(n) e[n \tau] \in S_{k+1 / 2}(N, \chi)$. Then it is verified that

$$
\Psi_{N, \chi, D}(f)(z)=\left(\overline{G^{\prime}\left(\chi_{1}, D\right)}\right)^{-1}\left\langle f, \Omega_{k, N}(-\bar{z}, * ; D, \chi)\right\rangle
$$

with $G^{\prime}\left(\chi_{1}, D\right)=\left(\frac{D}{N_{1}}\right) \chi_{1}(-D) W\left(\chi_{1}\right) \varepsilon^{1 / 2} N_{1}^{-k}$. By Theorem 2.2 and (1-3), we obtain that

$$
\begin{aligned}
\langle f, & \left.\Omega_{k, N}(-\bar{z}, * ; D, \chi)\right\rangle \\
& =\bar{C} \sum_{n=1}^{\infty} n^{k-1}\left(\sum_{d \mid n}\left(\frac{D}{d}\right) \chi(d)(n / d)^{k}\left\langle f, P_{k, N, n^{2}|D| / d^{2}, \chi}\right\rangle\right) \overline{e[-n \bar{z}]} \\
& =\bar{C} \sum_{n=1}^{\infty} n^{k-1}\left(\sum_{d \mid n}\left(\frac{D}{d}\right) \chi(d)(n / d)^{k} i_{4 N}^{-1} \frac{\Gamma(k-1 / 2)}{\left(4 \pi n^{2}|D| / d^{2}\right)^{k-1 / 2}} a\left(n^{2}|D| / d^{2}\right)\right) e[n z] \\
& =\overline{G^{\prime}\left(\chi_{1}, D\right)} \sum_{n=1}^{\infty}\left(\sum_{d \mid n}\left(\frac{D}{d}\right) \chi(d) d^{k-1} a\left(n^{2}|D| / d^{2}\right)\right) e[n z] .
\end{aligned}
$$

By (1-10) and [6, Proposition 1], we may confirm that $\overline{\Omega_{k, N}(-\bar{z}, \tau ; D, \chi)}$ belongs to $S_{2 k}\left(N, \chi^{2}\right)$ if $k \geqq 2$. We deduce the following theorem.

THEOREM 3.1. Suppose that $k \geqq 2$. Then

$$
\Psi_{N, \chi, D}(f)(z)=\sum_{n=1}^{\infty}\left(\sum_{d \mid n}\left(\frac{D}{d}\right) \chi(d) d^{k-1} a\left(n^{2}|D| / d^{2}\right)\right) e[n z]
$$

belongs to $S_{2 k}\left(N, \chi^{2}\right)$ for every $f(\tau)=\sum_{\varepsilon(-1)^{k} n \equiv 0,1(4)} a(n) e[n \tau] \in S_{k+1 / 2}(N, \chi)$, and the mapping $\Psi_{N, \chi, D}: S_{k+1 / 2}(N, \chi) \rightarrow S_{2 k}\left(N, \chi^{2}\right)$ has the kernel function $G^{\prime}\left(\chi_{1}, D\right)^{-1} \Omega_{k, N}(-\bar{z}, * ; D, \chi)$. Moreover, the diagram of mappings

$$
\begin{aligned}
& S_{k+1 / 2}(N, \chi) \stackrel{\Psi_{N, \chi, D}}{\longrightarrow} S_{2 k}\left(N, \chi^{2}\right) \\
& T_{k+1 / 2, N, \chi}\left(p^{2}\right) \quad\left(\text { resp. } \quad U\left(q^{2}\right)\right) \downarrow \quad \downarrow T_{2 k, N, \chi^{2}}(p) \quad\left(\text { resp. } \quad T_{2 k, N, \chi^{2}}(q)\right) \\
& S_{k+1 / 2}(N, \chi) \underset{\Psi_{N, \chi, D}}{\longrightarrow} S_{2 k}\left(N, \chi^{2}\right)
\end{aligned}
$$

is commutative for every prime $p, q(p \nmid N, q \mid N)$.

Proof 2. By virtue of Theorem 2.2, we obtain the equality (3-4). The relation (3-5) is an immediate consequence of a computation of Fourier coefficients. We omit the details.

We call the mapping $\Psi_{N, \chi, D}$ the $D^{t h}$ Shimura correspondence from $S_{k+1 / 2}(N, \chi)$ to $S_{2 k}\left(N, \chi^{2}\right)$. We denote by $\Psi_{N, \chi, D}^{*}$ the adjoint mapping of $\Psi_{N, \chi, D}$, i.e.,

$$
\left\langle f, \Psi_{N, \chi, D}^{*}(F)\right\rangle=\left\langle\Psi_{N, \chi, D}(f), F\right\rangle
$$


for every $f(\tau) \in S_{k+1 / 2}(N, \chi)$ and $F(z) \in S_{2 k}\left(N, \chi^{2}\right)$. It follows from this that

$$
\begin{aligned}
\Psi_{N, \chi, D}^{*}(F)(\tau) & \left(i_{N} G^{\prime}\left(\chi_{1}, D\right)\right)^{-1} \int_{\Gamma_{0}(N) \backslash \mathfrak{H}} F(z) \Omega_{k, N}(-\bar{z}, \tau ; D, \chi) y^{2 k} \frac{d x d y}{y^{2}} \\
= & \left(i_{N} G^{\prime}\left(\chi_{1}, D\right)\right)^{-1} i_{N} c_{k, D}^{-1} \sum_{\varepsilon(-1)^{k} m \equiv 0,1(4)} m^{k-1 / 2} \sum_{t \mid N_{2}} \mu(t)\left(\frac{D}{t}\right) \bar{\chi}_{1}(t) t^{k-1} \\
& \times\left(\int_{\Gamma_{0}(N) \backslash \mathfrak{H}} F(z) f_{k, N_{1}^{2}, N_{2} / t}\left(-t \bar{z} ; D, \varepsilon(-1)^{k} m, \chi_{1}\right) y^{2 k-2} d x d y\right) e[m \tau],
\end{aligned}
$$

where $z=x+i y \in \mathfrak{H}$. In particular, if $F$ is a new form of $S_{2 k}\left(N, \chi^{2}\right)$, then

$$
\begin{aligned}
& \Psi_{N, \chi, D}^{*}(F)(\tau)=c_{k, D}^{-1} G^{\prime}\left(\chi_{1}, D\right)^{-1} \sum_{\varepsilon(-1)^{k} m \equiv 0,1(4)} m^{k-1 / 2} \\
& \quad \times\left(\int_{\Gamma_{0}(N) \backslash \mathfrak{H}} F(z) f_{k, N_{1}^{2}, N_{2}}\left(-\bar{z} ; D, \varepsilon(-1)^{k} m, \chi_{1}\right) y^{2 k-2} d x d y\right) e[m \tau]
\end{aligned}
$$

For $D^{\prime} \in Z$, put

$$
L_{N_{1}^{2}, t, D}\left(D^{\prime}\right)=\left\{Q=\left(\begin{array}{cc}
a & b / 2 \\
b / 2 & c
\end{array}\right)\left|a, b, c \in \boldsymbol{Z}, N_{1}^{2} t\right| a, b^{2}-4 a c=N_{1}^{2} D D^{\prime}\right\} .
$$

For $t^{\prime} \in \boldsymbol{Z}(>0)$ and $Q \in L_{N_{1}^{2}, t, D}\left(D^{\prime}\right)$, put $Q \circ\left(\begin{array}{ll}t^{\prime} & 0 \\ 0 & 1\end{array}\right)={ }^{t}\left(\begin{array}{ll}t^{\prime} & 0 \\ 0 & 1\end{array}\right) Q\left(\begin{array}{ll}t^{\prime} & 0 \\ 0 & 1\end{array}\right)$. The mapping $Q \rightarrow Q \circ\left(\begin{array}{ll}t^{\prime} & 0 \\ 0 & 1\end{array}\right)$ gives a bijection of $L_{N_{1}^{2}, t, D}\left(D^{\prime}\right)$ onto $L_{N_{1}^{2}, t t^{\prime 2}, D}\left(D^{\prime} t^{\prime 2}\right)$. Since $\omega_{D}\left(Q \circ\left(\begin{array}{ll}t^{\prime} & 0 \\ 0 & 1\end{array}\right)\right)=\left(\frac{D}{t^{\prime 2}}\right) \omega_{D}(Q)$, we obtain

$$
f_{k, N_{1}^{2}, N_{2} / t}\left(t z ; D, \varepsilon(-1)^{k} m, \chi_{1}\right)=f_{k, N_{1}^{2}, t N_{2}}\left(z ; D, \varepsilon(-1)^{k} m t^{2}, \chi_{1}\right)
$$

for $t \quad\left(t \mid N_{2}\right)$. Hence we have

$$
\begin{aligned}
& \Psi_{N, \chi, D}^{*}(F)(\tau)=i_{N} c_{k, D}^{-1} G^{\prime}\left(\chi_{1}, D\right)^{-1} \sum_{\varepsilon(-1)^{k} m \equiv 0,1(4)} m^{k-1 / 2} \\
& \quad \times \sum_{t \mid N_{2}} \mu(t)\left(\frac{D}{t}\right) \bar{\chi}_{1}(t) t^{k-1}\left\langle F(z), f_{k, N_{1}^{2}, t N_{2}}\left(-z ; D, \varepsilon(-1)^{k} m t^{2}, \bar{\chi}_{1}\right)\right\rangle e[m \tau] .
\end{aligned}
$$

Note that

$$
f_{k, N_{1}^{2}, t N_{2}}\left(-z ; D, \varepsilon(-1)^{k} m t^{2}, \bar{\chi}_{1}\right)=f_{k, N_{1}^{2}, t N_{2}}\left(z ; D, \varepsilon(-1)^{k} m t^{2}, \bar{\chi}_{1}\right) .
$$

We now introduce an equivalence relation in $L_{N_{1}^{2}, N_{2} t, D}\left(\varepsilon(-1)^{k} m t^{2}\right)$ as follows. For $Q, Q^{\prime} \in L_{N_{1}^{2}, N_{2} t, D}\left(\varepsilon(-1)^{k} m t^{2}\right)$,

$$
Q \sim Q^{\prime} \text { if and only if } Q^{\prime}={ }^{t} g Q g \text { for some } g \in \Gamma_{0}(N) .
$$


Denote by $\Gamma_{0}(N) \backslash L_{N_{1}^{2}, N_{2} t, D}\left(\varepsilon(-1)^{k} m t^{2}\right)$ the set of all equivalence classes of $L_{N_{1}^{2}, N_{2} t, D}\left(\varepsilon(-1)^{k} m t^{2}\right)$ with respect to this equivalence relation. Then, we obtain

$$
\begin{aligned}
& L_{N_{1}^{2}, N_{2} t, D}\left(\varepsilon(-1)^{k} m t^{2}\right) \\
& \quad=\left\{{ }^{t} g \tilde{Q} g \mid g \in \Gamma_{\tilde{Q}} \backslash \Gamma_{0}(N), \tilde{Q} \in \Gamma_{0}(N) \backslash L_{N_{1}^{2}, N_{2} t, D}\left(\varepsilon(-1)^{k} m t^{2}\right)\right\},
\end{aligned}
$$

where $\Gamma_{\tilde{Q}}=\left\{g^{\prime} \in \Gamma_{0}(N) \mid{ }^{t} g^{\prime} \tilde{Q} g^{\prime}=\tilde{Q}\right\}$. For $Q=\left(\begin{array}{cc}a & b / 2 \\ b / 2 & c\end{array}\right)$, put $\omega_{D}(Q)=\omega_{D}(a, b, c)$, $Q(z, 1)=a z^{2}+b z+c=(z, 1) Q^{t}(z, 1)$ and $\bar{\chi}_{1}(Q)=\bar{\chi}_{1}(c)$. Then we see

$$
\begin{gathered}
\omega_{D}\left({ }^{t} g Q g\right)=\omega_{D}(Q), \quad \bar{\chi}_{1}(Q)=\bar{\chi}_{1}\left({ }^{t} g Q g\right) \bar{\chi}_{1}(\delta)^{2}, \\
Q(z, 1)=(N \gamma z+\delta)^{2}\left({ }^{t} g Q g\right)(g(z), 1)
\end{gathered}
$$

for every $g=\left(\begin{array}{cc}* & * \\ N \gamma & \delta\end{array}\right) \in \Gamma_{0}(N)$. This implies that

$$
\begin{aligned}
& f_{k, N_{1}^{2}, t N_{2}}\left(-z ; D, \varepsilon(-1)^{k} m t^{2}, \bar{\chi}_{1}\right) \\
& \quad=f_{k, N_{1}^{2}, t N_{2}}\left(z ; D, \varepsilon(-1)^{k} m t^{2}, \bar{\chi}_{1}\right) \\
& =\sum_{\tilde{Q} \in \Gamma_{0}(N) \backslash L_{N_{1}^{2}, N_{2} t, D}\left(\varepsilon(-1)^{k} m t^{2}\right)} \sum_{g} \bar{\chi}_{1}(\delta)^{2}(\gamma z+\delta)^{-2 k} \omega_{D}(\tilde{Q}) \tilde{Q}(g(z), 1)^{-k} \bar{\chi}_{1}(\tilde{Q}),
\end{aligned}
$$

where the sum $\sum_{g}$ is taken over all $g=\left(\begin{array}{ll}* & * \\ \gamma & \delta\end{array}\right) \in \Gamma_{\tilde{Q}} \backslash \Gamma_{0}(N)$. By [6, pp. 265-266], we may confirm

$$
\begin{aligned}
\left\langle F, \quad f_{k, N_{1}^{2}, t N_{2}}\left(-z ; D, \varepsilon(-1)^{k} m t^{2}, \bar{\chi}_{1}\right)\right\rangle \\
\quad=i_{N}^{-1} \pi\left(\begin{array}{c}
2 k-2 \\
k-1
\end{array}\right) 2^{-2 k+2}\left(|D| m t^{2}\right)^{1 / 2-k} \gamma_{k, N, \chi}\left(F ; D, \varepsilon(-1)^{k} m t^{2}\right)
\end{aligned}
$$

with

$$
\gamma_{k, N, \chi}\left(F ; D, \varepsilon(-1)^{k} m t^{2}\right)=\sum_{\tilde{Q} \in \Gamma_{0}(N) \backslash L_{N_{1}^{2}, N_{2} t, D}\left(\varepsilon(-1)^{k} m t^{2}\right)} \omega(\tilde{Q}) \chi_{1}(\tilde{Q}) \int_{c_{\tilde{Q}}} F(z) d_{\tilde{Q}, k} z,
$$

where $c_{\tilde{Q}}$ and $d_{\tilde{Q}, k} z$ is the symbol given in [6, p. 240]. Consequently, we obtain the following theorem.

THEOREM 3.2. Let $k$ be a positive integer such that $k \geqq 2$. Then

$$
\begin{aligned}
\Psi_{N, \chi, D}^{*}(F)(\tau)=c_{k, D}^{-1} G^{\prime}\left(\chi_{1}, D\right)^{-1} \pi\left(\begin{array}{c}
2 k-2 \\
k-1
\end{array}\right) 2^{-2 k+2}|D|^{1 / 2-k} \\
\times \sum_{\varepsilon(-1)^{k} m \equiv 0,1(4)} \sum_{t \mid N_{2}} \mu(t)\left(\frac{D}{t}\right) \bar{\chi}_{1}(t) t^{-k} \gamma_{k, N, \chi}\left(F ; D, \varepsilon(-1)^{k} m t^{2}\right) e[m \tau] .
\end{aligned}
$$


In particular, if $F$ is a new form of $S_{2 k}\left(N, \chi^{2}\right)$, then

$$
\begin{aligned}
\Psi_{N, \chi, D}^{*}(F)(\tau)= & c_{k, D}^{-1} G^{\prime}\left(\chi_{1}, D\right)^{-1}\left(\begin{array}{c}
2 k-2 \\
k-1
\end{array}\right) 2^{-2 k+2} \pi|D|^{1 / 2-k} \\
& \times \sum_{\varepsilon(-1)^{k} m \equiv 0,1(4)} \gamma_{k, N, \chi}\left(F ; D, \varepsilon(-1)^{k} m\right) e[m \tau] .
\end{aligned}
$$

Cycle integrals of this type were first investigated by Shintani [18].

4. Fourier coefficients of modular forms of half integral weight. This section is devoted to deriving a relation between Fourier coefficients of modular forms of half integral weight belonging to Kohnen's spaces and the critical values of zeta functions of modular forms. We first deduce the following

THEOREM 4.1. Let $m, n$ be positive integers such that $\varepsilon(-1)^{k} m, \varepsilon(-1)^{k} n \equiv 0,1$ $(\bmod 4), \varepsilon(-1)^{k} n$ is a fundamental discriminant and $(n, N)=1$. Suppose that $f(\tau)=$ $\sum_{\varepsilon(-1)^{k} n \equiv, 1(4)} a(n) e[n \tau] \in S_{k+1 / 2}(N, \chi)$ satisfies

$$
T_{k+1 / 2, N, \chi}\left(p^{2}\right)(f)=\lambda(p) f, \quad U\left(q^{2}\right) f=\lambda(q) f
$$

for every prime $p$ and $q(p \nmid N, q \mid N)$ and $\Psi_{N, \chi, D}(f)$ is a new form in $S_{2 k}\left(N, \chi^{2}\right)$. Let $F(z)=\sum_{n=1}^{\infty} a^{\prime}(n) e[n z]$ be the primitive form associated with $\Psi_{N, \chi, D}(f)$. Moreover, suppose that $f$ satisfies the following condition:

$$
\begin{aligned}
& \text { If } f^{\prime} \in S_{k+1 / 2}(N, \chi) \text { satisfies } T_{k+1 / 2, N, \chi}\left(p^{2}\right)\left(f^{\prime}\right)=\lambda(p) f^{\prime} \text { for every } \\
& \text { prime } p(p \nmid N) \text {, then there is a constant } c^{\prime} \in C \text { such that } f^{\prime}=c^{\prime} f .
\end{aligned}
$$

Then we have

$$
a(m) \overline{a(n)}\langle f, f\rangle^{-1}=\tilde{\tilde{c}} \gamma_{k, N, \chi}\left(F ; \varepsilon(-1)^{k} n, \varepsilon(-1)^{k} m\right)\langle F, F\rangle^{-1} .
$$

with

$$
\tilde{\tilde{c}}=c_{k, D}^{-1} G^{\prime}\left(\chi_{1}, D\right)^{-1} \pi\left(\begin{array}{c}
2 k-2 \\
k-1
\end{array}\right) 2^{-2 k+2}|D|^{1 / 2-k} .
$$

Proof 3. By Theorem 3.1, we have

$$
T_{2 k, N, \chi^{2}}(p) \Psi_{N, \chi, D}(f)=\lambda(p) \Psi_{N, \chi, D}(f) \text { for every prime } p(p \nmid N) .
$$

Since $\Psi_{N, \chi, D}(f)$ is a new form, there is a constant $c^{\prime \prime} \in \boldsymbol{C}$ such that

$$
\Psi_{N, \chi, D}(f)=c^{\prime \prime} F \text {. }
$$

Put $D=\varepsilon(-1)^{k} n$. Then, comparing Fourier coefficients of right and left hand sides at $e[z]$, we have

$$
a(|D|)=c^{\prime \prime} .
$$

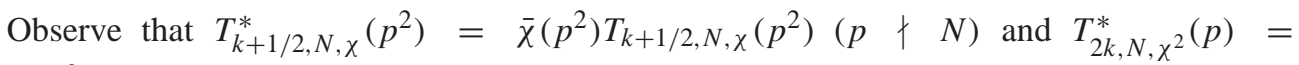
$\bar{\chi}\left(p^{2}\right) T_{2 k, N, \chi^{2}}(p)(p \nmid N)$ and $\Psi_{N, \chi, D}^{*}$ is the adjoint mapping of $\Psi_{N, \chi, D}$ with respect to 
the Petersson inner products. By Theorem 3.1, $\Psi_{N, \chi, D}$ is commutative with the action of Hecke operators. This implies that

$$
T_{k+1 / 2, N, \chi}\left(p^{2}\right) \Psi_{N, \chi, D}^{*}(F)=\lambda(p) \Psi_{N, \chi, D}^{*}(F) \text { for every prime } p(p \nmid N) .
$$

By the assumption, we obtain

$$
\Psi_{N, \chi, D}^{*}(F)=c^{\prime} f \text { for some } c^{\prime} \in C .
$$

Comparing Fourier coefficients of the both sides at $e[m \tau]$, we find that

$$
c^{\prime} a(m)=c_{k, D}^{-1} G^{\prime}\left(\chi_{1}, D\right)^{-1} \pi\left(\begin{array}{c}
2 k-2 \\
k-1
\end{array}\right) 2^{-2 k+2}|D|^{1 / 2-k} \gamma_{k, N, \chi}\left(F ; D, \varepsilon(-1)^{k} m\right) .
$$

On the other hand the value $c^{\prime}$ is determined as follows.

$$
\begin{aligned}
c^{\prime} a(m)\langle f, f\rangle & =a(m)\left\langle c^{\prime} f, f\right\rangle=a(m)\left\langle\Psi_{N, \chi, D}^{*}(F), f\right\rangle \\
& =a(m) \overline{\left\langle\Psi_{N, \chi, D}(f), F\right\rangle}=a(m) \overline{a(|D|)}\langle F, F\rangle .
\end{aligned}
$$

Hence we deduce that

$$
a(m) \overline{a(|D|)}\langle f, f\rangle^{-1}=\tilde{\tilde{c}} \gamma_{k, N, \chi}\left(F ; D, \varepsilon(-1)^{k} m\right)\langle F, F\rangle^{-1} .
$$

Since $D=\varepsilon(-1)^{k} n$, we see that $|D|=|n|=n$. This completes our proof.

Let $D$ be a fundamental discriminant such that $\varepsilon(-1)^{k} D>0$. Under the same assumption of Theorem 4.1, we have

$$
|a(|D|)|^{2}\langle f, f\rangle^{-1}=\tilde{\tilde{c}} \gamma_{k, N, \chi}(F ; D, D)\langle F, F\rangle^{-1}
$$

with

$$
\gamma_{k, N, \chi}(F ; D, D)=\sum_{\tilde{Q} \in \Gamma_{0}(N) \backslash L_{N_{1}^{2}, N_{2}, D}(D)} \omega_{D}(\tilde{Q}) \chi_{1}(\tilde{Q}) \int_{c_{\tilde{Q}}} F(z) d_{\tilde{Q}, k} z .
$$

In the remainder of this section, we assume that

$$
(D, N)=1 \quad \text { and } \quad\left(N_{1}, N_{2}\right)=1 .
$$

For $l\left(l \| N_{2}\right)$, we put

$$
W_{l}=\left(\begin{array}{cc}
\alpha l & \beta \\
\gamma N & \delta l
\end{array}\right) \quad \text { with } \alpha, \beta, \gamma, \delta \in Z \text { and } \alpha \delta l^{2}-\beta \gamma N=l,
$$

where $l \| N_{2}$ means $l \mid N_{2}$ and $\left(l, N_{2} / l\right)=1$. We determine $Q \mid W_{l}=(1 / l)^{t} W_{l} Q W_{l}$ for every $Q \in L_{N_{1}^{2}, N_{2}, D}(D)$. Since $W_{l}^{-1} g W_{l} \in \Gamma_{0}(N)$ for every $g \in \Gamma_{0}(N)$, we have the bijection of $\Gamma_{0}(N) \backslash L_{N_{1}^{2}, N_{2}, D}(D)$ onto $\Gamma_{0}(N) \backslash L_{N_{1}^{2}, N_{2}, D}(D)$. By [3, Section 1], we can take as a complete set of representatives for $\Gamma_{0}(N) \backslash L_{N_{1}^{2}, N_{2}, D}(D)$ the following set:

$$
\left\{\left(\begin{array}{cc}
0 & D N_{1} / 2 \\
D N_{1} / 2 & \mu
\end{array}\right)\left|W_{l}\right| \mu \in \boldsymbol{Z} / D N_{1} \boldsymbol{Z} \text { and } l \| N_{2}\right\} .
$$


For $\tilde{Q}=\left(\begin{array}{cc}a & b / 2 \\ b / 2 & c\end{array}\right) \in \Gamma_{0}(N) \backslash L_{N_{1}^{2}, N_{2}, D}(D), W_{l}=\left(\begin{array}{cc}\alpha l & \beta \\ \gamma N & \delta l\end{array}\right)\left(\delta=\delta_{l}\right)$, we obtain

$$
\chi_{1}\left(\tilde{Q} \mid W_{l}\right)=\chi_{1}(c) \chi_{1}\left(\delta^{2} l\right)=\chi_{1}(\tilde{Q}) \chi_{1}\left(\delta^{2} l\right), \omega_{D}\left(\tilde{Q} \mid W_{l}\right)=\omega_{D}(\tilde{Q})\left(\frac{D}{l}\right) .
$$

We note that if $l l^{\prime} \| N_{2},\left(l, l^{\prime}\right)=1$ and $p^{\alpha} \| N_{2}$, then

$$
\begin{gathered}
\delta_{l l^{\prime}} \equiv \delta_{l} \delta_{l^{\prime}} \quad(\bmod N), \quad \delta_{p^{\alpha}} \equiv 1 \quad\left(\bmod \left(N / p^{\alpha}\right)^{2}\right), \\
F(z) d_{Q \mid W_{l}, k} z=\left.F\right|_{2 k} W_{l}^{-1}\left(z^{\prime}\right) d_{Q, k} z^{\prime},
\end{gathered}
$$

where $z^{\prime}=W_{l}(z),\left.F\right|_{2 k} W_{l}^{-1}\left(z^{\prime}\right)=\left(\operatorname{det} W_{l}^{-1}\right)^{k}(c z+d)^{-2 k} F\left(W_{l}^{-1}\left(z^{\prime}\right)\right)$ with $W_{l}^{-1}=\left(\begin{array}{ll}* & * \\ c & d\end{array}\right)$. Since $F(z)$ is a primitive form, there are a constant $c(l)$ and a primitive form $F_{l}$ in $S_{2 k}\left(N, \chi^{2}\right)$ such that

$$
\left.F\right|_{2 k} W_{l}^{-1}=c(l) F_{l} .
$$

The constant $c(l)$ has the following property:

$$
\text { If } l l^{\prime} \| N_{2} \quad \text { and } \quad\left(l, l^{\prime}\right)=1 \text {, then } \quad c\left(l l^{\prime}\right)=c(l) c\left(l^{\prime}\right) .
$$

Put $F_{l}(z)=\sum_{n=1}^{\infty} a_{l}^{\prime}(n) e[n z]$. By (4-12) and (4-14), we find that

$$
\begin{aligned}
\gamma_{k, N, \chi}(F ; D, D)= & i\left(i D N_{1}\right)^{k-1} \sum_{l \| N_{2}}\left(\frac{D}{l}\right) \chi_{1}\left(\delta_{l}^{2} l\right) c(l) \\
& \times \sum_{n=1}^{\infty} a_{l}^{\prime}(n) \sum_{\mu \in \boldsymbol{Z} / N_{1} D \boldsymbol{Z}}\left(\frac{D}{\mu}\right) \chi_{1}(\mu) e_{D N_{1}}(-n \mu) \frac{\Gamma(k)}{(2 \pi n)^{k}} .
\end{aligned}
$$

We can easily check that

$$
\begin{aligned}
& \sum_{\mu \in \boldsymbol{Z} / N_{1} D \boldsymbol{Z}}\left(\frac{D}{\mu}\right) \chi_{1}(\mu) e_{D N_{1}}(-n \mu) \\
& \quad=\left(\frac{D}{N_{1}}\right) \chi_{1}(D)\left(\frac{D}{-1}\right)^{3 / 2}|D|^{1 / 2}\left(\frac{D}{n}\right) \bar{\chi}_{1}(n) \varepsilon W\left(\chi_{1}\right)
\end{aligned}
$$

and

$$
\begin{aligned}
L\left(s, F_{l},\left(\frac{D}{*}\right) \bar{\chi}_{1}\right) & =\sum_{n=1}^{\infty}\left(\frac{D}{n}\right) \bar{\chi}_{1}(n) a_{l}^{\prime}(n) n^{-s} \\
& =\prod_{p \mid l}\left(\frac{1-\left(\frac{D}{p}\right) \bar{\chi}_{1}(p) a^{\prime}(p) p^{-s}}{1-\left(\frac{D}{p}\right) \bar{\chi}_{1}(p) a_{l}^{\prime}(p) p^{-s}}\right) L\left(s, F,\left(\frac{D}{*}\right) \bar{\chi}_{1}\right) .
\end{aligned}
$$


By [2, Th. 4.6.16], we see that $a_{l}^{\prime}(p)=\chi^{2}(p) \overline{a^{\prime}(p)}$. Therefore we may deduce that

$$
\begin{aligned}
& \gamma_{k, N, \chi}(F ; D, D) \\
& =\frac{i\left(i D N_{1}\right)^{k-1}(k-1) !}{(2 \pi)^{k}}\left(\frac{D}{N_{1}}\right) \chi_{1}(D) W\left(\chi_{1}\right)\left(\frac{D}{-1}\right)^{3 / 2}|D|^{1 / 2} \varepsilon \sum_{l \| N_{2}}\left(\frac{D}{l}\right) \chi_{1}\left(\delta_{l}^{2} l\right) c(l) \\
& \quad \times \prod_{p \mid l}\left(\frac{1-\left(\frac{D}{p}\right) \bar{\chi}_{1}(p) a^{\prime}(p) p^{-k}}{1-\left(\frac{D}{p}\right) \bar{\chi}_{1}(p) a^{\prime}(p) p^{-k}}\right) L\left(k, F,\left(\frac{D}{*}\right) \bar{\chi}_{1}\right) .
\end{aligned}
$$

Put $N_{1}=\prod_{i=1}^{l} p_{i}^{v_{i}}, N_{2}=\prod_{i=l+1}^{s} p_{i}^{v_{i}}$. Then, by (4-16), (4-18) and (4-20), the summation taken over all $l$ in (4-21) is equal to

$$
\prod_{i=l+1}^{s}\left(1+\left(\frac{D}{p_{i}^{v_{i}}}\right) \chi_{1}\left(p_{i}^{\nu_{i}}\right) c\left(p_{i}^{\nu_{i}}\right)\left(\frac{1-\left(\frac{D}{p_{i}}\right) \bar{\chi}_{1}\left(p_{i}\right) a^{\prime}\left(p_{i}\right) p_{i}^{-k}}{\overline{1-\left(\frac{D}{p_{i}}\right) \bar{\chi}_{1}\left(p_{i}\right) a^{\prime}\left(p_{i}\right) p_{i}^{-k}}}\right)\right) .
$$

Consequently, we conclude the following theorem.

THEOREM 4.2. Under the same assumption as that of Theorem 4.1, suppose that $D$ is a fundamental discriminant such that $\varepsilon(-1)^{k} D>0,(N, D)=1$ and $\left(N_{1}, N_{2}\right)=1$. Then

$$
|a(|D|)|^{2}\langle F, F\rangle=R|D|^{k-1 / 2} N_{1}^{2 k-1}(k-1) ! \pi^{-k}\langle f, f\rangle L\left(k, F,\left(\frac{D}{*}\right) \bar{\chi}_{1}\right)
$$

with

$$
R=\prod_{i=l+1}^{s}\left(1+\left(\frac{D}{p_{i}^{v_{i}}}\right) \chi_{1}\left(p_{i}^{v_{i}}\right) c\left(p_{i}^{v_{i}}\right)\left(\frac{1-\left(\frac{D}{p_{i}}\right) \bar{\chi}_{1}\left(p_{i}\right) a^{\prime}\left(p_{i}\right) p_{i}^{-k}}{1-\left(\frac{D}{p_{i}}\right) \bar{\chi}_{1}\left(p_{i}\right) a^{\prime}\left(p_{i}\right) p_{i}^{-k}}\right)\right)
$$

It should be remarked that there are non-trivial examples satisfying the condition (4-2). By [11, Theorem 2.1], we may check that $\operatorname{dim} S_{5 / 2}(7, \chi)=\operatorname{dim} S_{4}\left(7, \chi^{2}\right)=1$. Hence, we see that a non-zero form $f$ in $S_{5 / 2}(7, \chi)$ provides such an example with $\chi^{2} \neq 1$.

\section{REFERENCES}

[ 1 ] N. Abramowitz and I. Stegun, Handbook of mathematical functions, New York, Dover, 1965.

[2] T. MiYaKe, Modular forms, Springer, Berlin, 1989.

[3] B. Gross, W. Kohnen and D. Zagier, Heegner points and derivatives of L series II, Math. Ann. 278 (1987), 497-562.

[ 4 ] W. Kohnen And D. ZAGier, Values of $L$-series of modular forms at the center of the critical strip, Invent. Math. 64 (1981), 175-198.

[ 5 ] W. Kohnen, New forms of half integral-integral weight, J. Reine Angew. Math. 333 (1982), 32-72.

[ 6 ] W. KoHnEn, Fourier coefficients of modular forms of half-integral weight, Math. Ann. 271 (1985), 237-268.

[ 7 ] W. Kohnen, A remark on the Shimura correspondence, Glasg. Math. J. 30 (1988), 285-291.

[ 8 ] H. KojIma, Remark on Fourier coefficients of modular forms of half integral weight belonging to Kohnen's spaces, J. Math. Soc. Japan 51 (1999), 715-730. 
[ 9 ] H. KojIma, Remark on Fourier coefficients of modular forms of half integral weight belonging to Kohnen's spaces II, Kodai Math. J. 22 (1999), 99-115.

[10] H. KoJima, On the Fourier coefficients of Maass wave forms of half integral weight over an imaginary quadratic fields, J. Reine Angew. Math. 526 (2000), 155-179.

[11] H. Kojima, Remark on the dimension of Kohnen's spaces of half integral weight with square free level, Proc. Japan Acad. Ser. A Math. Sci. 78 (2002), 18-21.

[12] K. K. MAKDISI, On the Fourier coefficients of non holomorphic Hilbert modular forms of half integral weight, Duke Math. J. 84 (1996), 399-452.

[13] M. Manickam, B. Ramakrishnan and T. C. Vasudevan, On Shintani correspondence, Proc. Indian Acad. Sci. Math. Sci. 99 (1989), 235-247.

[14] M. Manickam and B. Ramakrishnan, On Shimura, Shintani and Eichler-Zagier correspondence, Trans. Amer. Math. Soc. 352 (2000), 2601-2617.

[15] H. SAKATA, On the Kohnen-Zagier formula in higher multiplicity cases-The case of general level, preprint.

[16] G. Shimura, On modular forms of half-integral weight, Ann. of Math. (2) 97 (1973), 440-481.

[17] G. ShImURA, On the Fourier coefficients of Hilbert modular forms of half-integral weight, Duke Math. J. 71 (1993), 501-557.

[18] T. Shintani, On construction of holomorphic cusp forms of half-integral weight, Nagoya Math. J. 58 (1975), 83-126.

[19] M. UEDA, The decomposition of the space of cusp forms of half-integral weight and trace formula of Hecke operators, J. Math. Kyoto Univ. 28 (1988), 505-555.

[20] J.-L. Waldspurger, Sur les coefficients de Fourier des formes modulaires de poids demi-entier, J. Math. Pures Appl. 60 (1981), 375-484.

DEPARTMENT OF MATHEMATICS

FACULTY OF EDUCATION

IWATE UNIVERSITY, MORIOKA 020-8550

JAPAN

E-mail address: kojima@iwate-u.ac.jp
Miyagi National College of Technology

Medeshima Shiote Nodayama 48

NATORI CITY 981-1239

JAPAN

E-mail address: tokuno@miyagi-ct.ac.jp 